ANNALES

POLONICI MATHEMATICI

$98.1(2010)$

\title{
Multiple solutions for a class of $p(x)$-Laplacian equations involving the critical exponent
}

\author{
by Xing Zhang, Xia Zhang and Yongqiang Fu (Harbin)
}

\begin{abstract}
We study the multiplicity of solutions for a class of $p(x)$-Laplacian equations involving the critical exponent. Under suitable assumptions, we obtain a sequence of radially symmetric solutions associated with a sequence of positive energies going toward infinity.
\end{abstract}

1. Introduction and main results. With the emergence of nonlinear problems in natural science and engineering, the previous studies made in Sobolev spaces demonstrate their limitations in applications. For example, a class of nonlinear problems with variable exponential growth is a new research field and reflects new kinds of physical phenomena. In the studies of nonlinear problems, variable exponent Sobolev spaces play an important role.

In recent years, differential equations and variational problems with $p(x)$ growth conditions have been studied extensively (see for example 1, 2, 3, 6, 13, 15]). In [9], Fu established a principle of concentration compactness in the Sobolev space $W^{1, p(x)}$, which is a generalization of the corresponding result in [12]; he also discussed the existence of solutions for a class of $p(x)$ Laplacian equations with critical growth.

In this paper, we will consider multiple radial solutions for the following kind of $p(x)$-Laplacian equations with critical exponent:

$$
\begin{cases}-\operatorname{div}\left(|\nabla u|^{p(x)-2} \nabla u\right)+|u|^{p(x)-2} u=|u|^{p^{*}(x)-2} u+f(x, u), & x \in \Omega \\ u(x)=0, & x \in \partial \Omega\end{cases}
$$

where $\Omega \subset \mathbb{R}^{N}$ is a bounded, radially symmetric domain and $0 \notin \bar{\Omega}, p$ is Lipschitz continuous, radially symmetric on $\bar{\Omega}$ and satisfies $1<p_{-} \leq p(x) \leq$

2010 Mathematics Subject Classification: Primary 35J60.

Key words and phrases: $p(x)$-Laplacian equation, variable exponent Sobolev space, multiple solution. 
$p_{+}<N$, and $p^{*}(x)=N p(x) /(N-p(x))$. Here we denote

$$
p_{+}=\sup _{x \in \bar{\Omega}} p(x), \quad p_{-}=\inf _{x \in \bar{\Omega}} p(x)
$$

and write $p_{1}(x) \ll p_{2}(x)$ whenever $\inf _{x \in \bar{\Omega}}\left(p_{2}(x)-p_{1}(x)\right)>0$. Throughout this paper, we assume that $f(x, t)$ satisfies the following conditions:

(H1) $f \in C(\bar{\Omega} \times \mathbb{R}, \mathbb{R})$ and there exists $\alpha \in C(\bar{\Omega})$ with either $1<\alpha_{-} \leq$ $\alpha(x) \leq p(x)$ for all $x \in \bar{\Omega}$, or $p(x) \leq \alpha(x) \ll p^{*}(x)$ for all $x \in \bar{\Omega}$, and $a_{0}, a_{1}>0$ such that for any $(x, t) \in \Omega \times \mathbb{R}$,

$$
|f(x, t)| \leq a_{0}+a_{1}|t|^{\alpha(x)-1} .
$$

(H2) $f(x, t)=-f(x,-t)$ for any $(x, t) \in \Omega \times \mathbb{R}$.

(H3) $f(x, t)=f(|x|, t)$ for any $(x, t) \in \Omega \times \mathbb{R}$.

In this paper, we will prove the following result.

TheOREM 1.1. Assume hypotheses (H1)-(H3) are fulfilled. Then problem (1.1) has a sequence $\left\{u_{n}\right\} \subset W^{1, p(x)}(\Omega)$ of radially symmetric solutions such that

$$
\varphi\left(u_{n}\right)=\int_{\Omega}\left(\frac{\left|\nabla u_{n}\right|^{p(x)}+\left|u_{n}\right|^{p(x)}}{p(x)}-\frac{\left|u_{n}\right|^{p^{*}(x)}}{p^{*}(x)}-F\left(x, u_{n}\right)\right) d x \rightarrow \infty
$$

as $n \rightarrow \infty$, where $F(x, t)=\int_{0}^{t} f(x, s) d s$.

2. Preliminaries. First we recall some basic properties of variable exponent Lebesgue spaces $L^{p(x)}(\Omega)$ and variable exponent Sobolev spaces $W^{1, p(x)}(\Omega)$, where $\Omega \subset \mathbb{R}^{N}$ is a domain. For a deeper treatment of these spaces, we refer to [4, 5, 7, 8, 10].

Let $\mathbf{P}(\Omega)$ be the set of all Lebesgue measurable functions $p: \Omega \rightarrow[1, \infty)$ and

$$
|u|_{p(x)}=\inf \left\{\lambda>0: \int_{\Omega}|u / \lambda|^{p(x)} d x \leq 1\right\} .
$$

The variable exponent Lebesgue space $L^{p(x)}(\Omega)$ is the class of all functions $u$ such that $\int_{\Omega}|u(x)|^{p(x)} d x<\infty$. Under the assumption that $1 \leq p_{-} \leq p_{+}$ $<\infty, L^{p(x)}(\Omega)$ is a Banach space equipped with the norm 2.1 .

The variable exponent Sobolev space $W^{1, p(x)}(\Omega)$ is the class of all functions $u \in L^{p(x)}(\Omega)$ such that $|\nabla u| \in L^{p(x)}(\Omega)$ and it can be equipped with the norm

$$
\|u\|_{1, p(x)}=|u|_{p(x)}+|\nabla u|_{p(x)} .
$$

For $u \in W^{1, p(x)}(\Omega)$, if we define

$$
\|u\|=\inf \left\{\lambda>0: \int_{\Omega} \frac{|u|^{p(x)}+|\nabla u|^{p(x)}}{\lambda^{p(x)}} d x \leq 1\right\},
$$

then $\|u\|$ and $\|u\|_{1, p(x)}$ are equivalent norms on $W^{1, p(x)}(\Omega)$. 
By $W_{0}^{1, p(x)}(\Omega)$ we denote the subspace of $W^{1, p(x)}(\Omega)$ which is the closure of $C_{0}^{\infty}(\Omega)$ with respect to the norm 2.2 . We know that if $\Omega \subset \mathbb{R}^{N}$ is a bounded domain, $\|u\|_{1, p(x)}$ and $|\nabla u|_{p(x)}$ are equivalent norms on $W_{0}^{1, p(x)}(\Omega)$.

In this paper, we will discuss equation (1.1) when $p(x)$ is radially symmetric. First of all, let us introduce some notation.

Let $O(N)$ be the group of orthogonal linear transformations in $\mathbb{R}^{N}$, and $G$ be a subgroup of $O(N)$. For $x \neq 0$, we denote the cardinality of $G_{x}=$ $\{g x: x \in G\}$ by $\left|G_{x}\right|$ and set $|G|=\inf _{x \in \mathbb{R}^{N}, x \neq 0}\left|G_{x}\right|$. An open subset $\Omega$ of $\mathbb{R}^{N}$ is $G$-invariant if $g \Omega=\Omega$ for any $g \in G$.

Definition 2.1. Let $\Omega$ be a $G$-invariant open subset of $\mathbb{R}^{N}$. The action of $G$ on $W_{0}^{1, p(x)}(\Omega)$ is defined by

$$
g u(x)=u\left(g^{-1} x\right)
$$

for any $u \in W_{0}^{1, p(x)}(\Omega)$. The subspace of invariant functions is defined by

$$
W_{0, G}^{1, p(x)}(\Omega)=\left\{u \in W_{0}^{1, p(x)}(\Omega): g u=u, \forall g \in G\right\} .
$$

A functional $\varphi: W_{0}^{1, p(x)}(\Omega) \rightarrow \mathbb{R}$ is $G$-invariant if $\varphi \circ g=\varphi$ for any $g \in G$.

Under the condition $1<p_{-} \leq p_{+}<\infty, W_{0}^{1, p(x)}(\Omega)$ is a separable and reflexive Banach space, so $W_{0, G}^{1, p(x)}(\Omega)$ is also a separable and reflexive Banach space. Thus there exist $\left\{e_{n}\right\}_{n=1}^{\infty} \subset W_{0, G}^{1, p(x)}(\Omega)$ and $\left\{f_{m}\right\}_{m=1}^{\infty} \subset$ $\left(W_{0, G}^{1, p(x)}(\Omega)\right)^{*}$ such that

$$
f_{m}\left(e_{n}\right)= \begin{cases}1 & \text { if } n=m \\ 0 & \text { if } n \neq m\end{cases}
$$

and

$$
\begin{aligned}
W_{0, G}^{1, p(x)}(\Omega) & =\overline{\operatorname{span}\left\{e_{i}: i=1,2, \ldots\right\}}, \\
\left(W_{0, G}^{1, p(x)}(\Omega)\right)^{*} & =\overline{\operatorname{span}\left\{f_{j}: j=1,2, \ldots\right\}},
\end{aligned}
$$

where $\left(W_{0, G}^{1, p(x)}(\Omega)\right)^{*}$ is the dual of $W_{0, G}^{1, p(x)}(\Omega)$. In the following, we will denote $Y_{n}=\operatorname{span}\left\{e_{1}, \ldots, e_{n}\right\}, Z_{n}=\overline{\operatorname{span}\left\{e_{k}: k=n, \cdots\right\}}$, for any $n \in \mathbb{N}$.

In order to find critical points of (1.1), we will need the following result due to $\mathrm{Fu}[9$. Let $M(\bar{\Omega})$ denote the class of nonnegative Borel measures of finite total mass and let $\mu_{n} \rightarrow \mu$ in $M(\bar{\Omega})$ be defined by $\int_{\bar{\Omega}} \eta d \mu_{n} \rightarrow \int_{\bar{\Omega}} \eta d \mu$ for any $\eta \in C^{\infty}(\Omega) \cap C(\bar{\Omega})$.

Proposition 2.1. Let $\left\{u_{n}\right\}$ be a sequence in $W_{0}^{1, p(x)}(\Omega)$ with $\left|\nabla u_{n}\right|_{p(x)}$ $\leq 1$ such that $u_{n} \rightarrow u$ weakly in $W_{0}^{1, p(x)}(\Omega),\left|\nabla u_{n}\right|^{p(x)} \rightarrow \mu,\left|u_{n}\right|^{p^{*}(x)} \rightarrow \nu$ 
in $M(\bar{\Omega})$. Then the limit measures are of the form

$$
\begin{aligned}
& \mu=|\nabla u|^{p(x)}+\sum_{j \in J} \mu_{j} \delta_{x_{j}}+\widetilde{\mu}, \quad \mu(\bar{\Omega}) \leq 1, \\
& \nu=|u|^{p^{*}(x)}+\sum_{j \in J} \nu_{j} \delta_{x_{j}}, \quad \nu(\bar{\Omega}) \leq C^{*},
\end{aligned}
$$

where $C^{*}=\sup \left\{\int_{\Omega}|u|^{p^{*}(x)} d x: u \in W_{0}^{1, p(x)}(\Omega),|\nabla u|_{p(x)} \leq 1\right\}$, J is a countable set, and $\widetilde{\mu} \in M(\bar{\Omega})$ is a nonatomic positive measure. The atoms and the regular part satisfy the generalized Sobolev inequality

$$
\begin{aligned}
\nu(\bar{\Omega}) & \leq C^{*} \max \left\{\mu(\bar{\Omega})^{p_{+}^{*} / p_{-}}, \mu(\bar{\Omega})^{p_{-}^{*} / p_{+}}\right\}, \\
\nu_{j} & \leq C^{*} \max \left\{\mu_{j}^{p_{+}^{*} / p_{-}}, \mu_{j}^{p_{-}^{*} / p_{+}}\right\} .
\end{aligned}
$$

Lemma 2.2. Set $\theta_{n}=\sup _{u \in Z_{n},\|u\| \leq 1} \int_{\Omega}|u|^{p^{*}(x)} d x$. If $|G|=\infty$, then $\theta_{n} \rightarrow 0$ as $n \rightarrow \infty$.

Proof. It is obvious that $0 \leq \theta_{n+1} \leq \theta_{n}$, so $\theta_{n} \rightarrow \theta \geq 0$ as $n \rightarrow \infty$. There exist $u_{n} \in Z_{n}$ with $\left\|u_{n}\right\| \leq 1$ such that

$$
0 \leq \theta_{n}-\int_{\Omega}\left|u_{n}\right|^{p^{*}(x)} d x<\frac{1}{n}
$$

for each $n=1,2, \ldots$ As $W_{0, G}^{1, p(x)}(\Omega)$ is reflexive, passing to a subsequence, still denoted by $u_{n}$, we may assume that there exists $u \in W_{0, G}^{1, p(x)}(\Omega)$ such that $u_{n} \rightarrow u$ weakly in $W_{0, G}^{1, p(x)}(\Omega)$, as $n \rightarrow \infty$.

(i) $u=0$. In fact, for any $f_{m} \in\left\{f_{n}: n=1,2, \ldots\right\}$, we have $f_{m}\left(u_{n}\right)=0$ when $n>m$, so $f_{m}\left(u_{n}\right) \rightarrow 0$ as $n \rightarrow \infty$. It is immediate that $f_{m}(u)=0$ for any $m \in \mathbb{N}$. Since

$$
\left(W_{0, G}^{1, p(x)}(\Omega)\right)^{*}=\overline{\operatorname{span}\left\{f_{j}: j=1,2, \ldots\right\}},
$$

it follows that $u=0$.

By Proposition 2.1, there exist a finite measure $\nu$ and sequences $\left\{x_{j}\right\}$ $\subset \bar{\Omega}$ such that $\left|u_{n}\right|^{p^{*}(x)} \rightarrow \nu=\sum_{j \in J} \nu_{j} \delta_{x_{j}}$ in $M(\bar{\Omega})$, where $J$ is a countable set.

(ii) We claim $\nu_{j}=0$ for any $j \in J$. Suppose that there exists $j_{0} \in J$ such that $\nu_{j_{0}}=\nu\left(\left\{x_{j_{0}}\right\}\right)>0$. As $u_{n} \in W_{0, G}^{1, p(x)}(\Omega)$, the measure $\nu$ is $G$-invariant. For any $g \in G, \nu\left(\left\{g x_{j_{0}}\right\}\right)=\nu\left(\left\{x_{j_{0}}\right\}\right)>0$. As $|G|=\infty, \nu\left(\left\{g x_{j_{0}}: g \in G\right\}\right)=$ $\infty$. But we know the measure $\nu$ is finite, so that is a contradiction. Set $\eta \equiv 1$. Then we get $\int_{\bar{\Omega}}\left|u_{n}\right|^{p^{*}(x)} \eta d x \rightarrow \int_{\bar{\Omega}} \eta d \nu=0$. Hence it is easy to deduce the result. 


\section{The proof of Theorem 1.1}

Definition 3.1. We say that $u_{0} \in W_{0}^{1, p(x)}(\Omega)$ is a weak solution of problem (1.1) if for any $u \in W_{0}^{1, p(x)}(\Omega)$,

$$
\int_{\Omega}\left(\left|\nabla u_{0}\right|^{p(x)-2} \nabla u_{0} \nabla u+\left|u_{0}\right|^{p(x)-2} u_{0} u-\left|u_{0}\right|^{p^{*}(x)-2} u_{0} u-f\left(x, u_{0}\right) u\right) d x=0 .
$$

It is easy to check that $\varphi \in C^{1}\left(W_{0}^{1, p(x)}(\Omega), \mathbb{R}\right)$ and the weak solutions for problem (1.1) coincide with the critical points of $\varphi$.

In the following, we assume $G=O(N)$. By condition (H3), it is immediate to see that $\varphi$ is $O(N)$-invariant. Then, by the principle of symmetric criticality of Krawcewicz and Marzantowicz [11], we know that $u_{0}$ is a critical point of $\varphi$ if and only if $u_{0}$ is a critical point of $\widetilde{\varphi}=\left.\varphi\right|_{W_{0, O(N)}^{1, p(x)}(\Omega)}$. Therefore, it suffices to prove the existence of a sequence of critical points of $\widetilde{\varphi}$ on $W_{0, O(N)}^{1, p(x)}(\Omega)$.

Lemma 3.1. Any (PS) sequence $\left\{u_{n}\right\} \subset W_{0, O(N)}^{1, p(x)}(\Omega)$, i.e. $\left|\widetilde{\varphi}\left(u_{n}\right)\right| \leq c$ and $\widetilde{\varphi}^{\prime}\left(u_{n}\right) \rightarrow 0$, as $n \rightarrow \infty$, is bounded.

Proof. Let $\mu(x)=\left(p(x)+p^{*}(x)\right) / 2$. Denote

$$
l_{1}=\inf _{x \in \bar{\Omega}}\left(\frac{1}{p(x)}-\frac{1}{\mu(x)}\right)>0, \quad l_{2}=\inf _{x \in \bar{\Omega}}\left(\frac{1}{\mu(x)}-\frac{1}{p^{*}(x)}\right)>0 .
$$

Then we get

$$
\begin{aligned}
\widetilde{\varphi}\left(u_{n}\right) & -\left\langle\widetilde{\varphi}^{\prime}\left(u_{n}\right), u_{n} / \mu\right\rangle \\
= & \int_{\Omega}\left(\left(\frac{1}{p(x)}-\frac{1}{\mu(x)}\right)\left(\left|\nabla u_{n}\right|^{p(x)}+\left|u_{n}\right|^{p(x)}\right)+\frac{u_{n}}{\mu(x)^{2}}\left|\nabla u_{n}\right|^{p(x)-2} \nabla u_{n} \nabla \mu\right. \\
& \left.+\left(\frac{1}{\mu(x)}-\frac{1}{p^{*}(x)}\right)\left|u_{n}\right|^{p^{*}(x)}+\frac{1}{\mu(x)} f\left(x, u_{n}\right) u_{n}-F\left(x, u_{n}\right)\right) d x \\
\geq & \int_{\Omega}\left(l_{1}\left|\nabla u_{n}\right|^{p(x)}+l_{2}\left|u_{n}\right|^{p^{*}(x)}+\frac{u_{n}}{\mu(x)^{2}}\left|\nabla u_{n}\right|^{p(x)-2} \nabla u_{n} \nabla \mu\right. \\
& \left.+\frac{1}{\mu(x)} f\left(x, u_{n}\right) u_{n}-F\left(x, u_{n}\right)\right) d x .
\end{aligned}
$$

By condition (H1), for any $(x, t) \in \Omega \times \mathbb{R}$,

$$
|F(x, t)| \leq a_{0}|t|+a_{1} \frac{|t|^{\alpha(x)}}{\alpha(x)} .
$$

Hence there exist $c_{0}, c_{1}>0$ such that

$$
\left|\frac{1}{\mu(x)} f\left(x, u_{n}\right) u_{n}-F\left(x, u_{n}\right)\right| \leq c_{0}\left|u_{n}\right|+c_{1}\left|u_{n}\right|^{\alpha(x)} .
$$


As $\mu(x)$ is Lipschitz continuous on $\bar{\Omega}$, the Young inequality implies that for any $\varepsilon_{1} \in(0,1)$,

$$
\begin{aligned}
\left.\left|\frac{u_{n}}{\mu(x)^{2}}\right| \nabla u_{n}\right|^{p(x)-2} \nabla u_{n} \nabla \mu \mid & \leq c_{2}\left|\nabla u_{n}\right|^{p(x)-1}\left|u_{n}\right| \\
& \leq c_{2}\left(\frac{\varepsilon_{1}(p(x)-1)}{p(x)}\left|\nabla u_{n}\right|^{p(x)}+\frac{\varepsilon_{1}^{1-p(x)}}{p(x)}\left|u_{n}\right|^{p(x)}\right) \\
& \leq c_{2}\left(\varepsilon_{1}\left|\nabla u_{n}\right|^{p(x)}+\varepsilon_{1}^{1-p_{+}}\left|u_{n}\right|^{p(x)}\right) .
\end{aligned}
$$

Let $\varepsilon_{1}$ be so small that $c_{2} \varepsilon_{1} \leq l_{1} / 2$. Then

$$
\begin{aligned}
& \tilde{\varphi}\left(u_{n}\right)-\left\langle\widetilde{\varphi}^{\prime}\left(u_{n}\right), u_{n} / \mu(x)\right\rangle \\
& \geq \int_{\Omega}\left(\frac{l_{1}}{2}\left|\nabla u_{n}\right|^{p(x)}+l_{2}\left|u_{n}\right|^{p^{*}(x)}-c_{0}\left|u_{n}\right|-c_{1}\left|u_{n}\right|^{\alpha(x)}-c_{2} \varepsilon_{1}^{1-p_{+}}\left|u_{n}\right|^{p(x)}\right) d x .
\end{aligned}
$$

Noting that $\alpha(x) \ll p^{*}(x), p(x) \ll p^{*}(x)$, by the Young inequality, for any $\varepsilon_{2}, \varepsilon_{3}, \varepsilon_{4} \in(0,1)$, we get

$$
\begin{aligned}
\left|u_{n}\right| & \leq \frac{\varepsilon_{2}\left|u_{n}\right|^{p^{*}(x)}}{p^{*}(x)}+\frac{p^{*}(x)-1}{p^{*}(x)} \varepsilon_{2}^{\frac{1}{1-p^{*}(x)}} \\
& \leq \varepsilon_{2}\left|u_{n}\right|^{p^{*}(x)}+\varepsilon_{2}^{-\frac{1}{p_{-}^{*}-1}}, \\
\left|u_{n}\right|^{\alpha(x)} & \leq \frac{\varepsilon_{3} \alpha(x)\left|u_{n}\right|^{p^{*}(x)}}{p^{*}(x)}+\frac{p^{*}(x)-\alpha(x)}{p^{*}(x)} \varepsilon_{3}^{\frac{\alpha(x)}{\alpha(x)-p^{*}(x)}} \\
& \leq \varepsilon_{3}\left|u_{n}\right|^{p^{*}(x)}+\varepsilon_{3}^{-\frac{\alpha+}{\left(p^{*}-\alpha\right)_{-}}}, \\
\left|u_{n}\right|^{p(x)} & \leq \frac{\varepsilon_{4} p(x)}{p^{*}(x)}\left|u_{n}\right|^{p^{*}(x)}+\frac{p^{*}(x)-p(x)}{p^{*}(x)} \varepsilon_{4}^{\frac{p(x)}{p(x)-p^{*}(x)}} \\
& \leq \varepsilon_{4}\left|u_{n}\right|^{p^{*}(x)}+\varepsilon_{4}^{-\frac{p+}{\left(p^{*}-p\right)_{-}}} .
\end{aligned}
$$

Let $\varepsilon_{2}, \varepsilon_{3}, \varepsilon_{4}$ be so small that $c_{0} \varepsilon_{2}+c_{1} \varepsilon_{3}+c_{2} \varepsilon_{1}^{1-p_{+}} \varepsilon_{4} \leq l_{2}$. Then

$$
\widetilde{\varphi}\left(u_{n}\right)-\left\langle\widetilde{\varphi}^{\prime}\left(u_{n}\right), u_{n} / \mu\right\rangle \geq \int_{\Omega}\left(\frac{l_{1}}{2}\left|\nabla u_{n}\right|^{p(x)}-c_{3}\right) d x .
$$

Note that

$$
\begin{aligned}
\left|\left\langle\widetilde{\varphi}^{\prime}\left(u_{n}\right), u_{n} / \mu\right\rangle\right| & \leq\left\|\widetilde{\varphi}^{\prime}\left(u_{n}\right)\right\| \cdot\left\|u_{n} / \mu\right\| \\
& \leq c_{4}\left\|\widetilde{\varphi}^{\prime}\left(u_{n}\right)\right\| \cdot\left|\nabla\left(u_{n} / \mu\right)\right|_{p(x)} \leq c_{5}\left\|\widetilde{\varphi}^{\prime}\left(u_{n}\right)\right\| \cdot\left|\nabla u_{n}\right|_{p(x)},
\end{aligned}
$$

and for $n \in \mathbb{N}$ large enough, we have $c_{5}\left\|\varphi^{\prime}\left(u_{n}\right)\right\| \leq l_{1} / 4$. It is easy to show that if $\left|\nabla u_{n}\right|_{p(x)} \geq 1$, then

$$
\left|\nabla u_{n}\right|_{p(x)} \leq \int_{\Omega}\left|\nabla u_{n}\right|^{p(x)} d x
$$


and thus we get

$$
\varphi\left(u_{n}\right) \geq \int_{\Omega}\left(\frac{l_{1}}{4}\left|\nabla u_{n}\right|^{p(x)}-c_{3}\right) d x .
$$

Hence $\left|\nabla u_{n}\right|_{p(x)}$ is bounded. If $\left|\nabla u_{n}\right|_{p(x)}<1$, it is immediate that $\left\{u_{n}\right\}$ is bounded. Thus we get the result.

LEMMA 3.2. Any (PS) sequence contains a convergent subsequence.

Proof. Let $\left\{u_{n}\right\} \subset W_{0, O(N)}^{1, p(x)}(\Omega)$ be a (PS) sequence. By Lemma 3.1, $\left\{u_{n}\right\}$ is bounded. As $W_{0, O(N)}^{1, p(x)}(\Omega)$ is reflexive, passing to a subsequence, still denoted by $\left\{u_{n}\right\}$, we may assume that there exists $u \in W_{0, O(N)}^{1, p(x)}(\Omega)$ such that $u_{n} \rightarrow u$ weakly in $W_{0, O(N)}^{1, p(x)}(\Omega)$. We can also achieve that $u_{n} \rightarrow u$ weakly in $W_{0}^{1, p(x)}(\Omega)$. Then $u_{n} \rightarrow u$ in $L^{p(x)}(\Omega)$ and in $L^{\alpha(x)}(\Omega)$, as $n \rightarrow \infty$.

Note that

$$
\begin{aligned}
& \left\langle\widetilde{\varphi}^{\prime}\left(u_{n}\right)-\widetilde{\varphi}^{\prime}(u), u_{n}-u\right\rangle \\
& =\int_{\Omega}\left(\left(\left|\nabla u_{n}\right|^{p(x)-2} \nabla u_{n}-|\nabla u|^{p(x)-2} \nabla u\right) \nabla\left(u_{n}-u\right)\right. \\
& \quad+\left(\left|u_{n}\right|^{p(x)-2} u_{n}-|u|^{p(x)-2} u\right)\left(u_{n}-u\right) \\
& \left.\quad-\left(\left|u_{n}\right|^{p^{*}(x)-2} u_{n}-|u|^{p^{*}(x)-2} u\right)\left(u_{n}-u\right)-\left(f\left(x, u_{n}\right)-f(x, u)\right)\left(u_{n}-u\right)\right) d x .
\end{aligned}
$$

It is easy to deduce that

$$
\begin{gathered}
\left\langle\widetilde{\varphi}^{\prime}\left(u_{n}\right)-\widetilde{\varphi}^{\prime}(u), u_{n}-u\right\rangle \rightarrow 0, \\
\int_{\Omega}\left(\left|u_{n}\right|^{p(x)-2} u_{n}-|u|^{p(x)-2} u\right)\left(u_{n}-u\right) d x \rightarrow 0 .
\end{gathered}
$$

By Proposition 2.1, there exist a finite measure $\nu$ and a sequence $\left\{x_{j}\right\} \subset \bar{\Omega}$ such that $\left|u_{n}-u\right|^{p^{*}(x)} \rightarrow \nu=\sum_{j \in J} \nu_{j} \delta_{x_{j}}$ in $M(\bar{\Omega})$, where $J$ is a countable set. Note that $|O(N)|=\infty$, so as in (ii) of the proof of Lemma 2.2, we get $\nu=0$. Set $\eta \equiv 1$, so that

$$
\int_{\bar{\Omega}}\left|u_{n}-u\right|^{p^{*}(x)} \eta d x \rightarrow \int_{\bar{\Omega}} \eta d \nu=0
$$

i.e. $u_{n} \rightarrow u$ in $L^{p^{*}(x)}(\Omega)$, as $n \rightarrow \infty$. Then

$$
\int_{\Omega}\left(\left|u_{n}\right|^{p^{*}(x)-2} u_{n}-|u|^{p^{*}(x)-2} u\right)\left(u_{n}-u\right) d x \rightarrow 0
$$

as $n \rightarrow \infty$. By condition (H1), we obtain

$$
\begin{aligned}
\int_{\Omega}\left|f\left(x, u_{n}\right)\left(u_{n}-u\right)\right| d x & \leq \int_{\Omega}\left(a_{0}+a_{1}\left|u_{n}\right|^{\alpha(x)-1}\right)\left|u_{n}-u\right| d x \\
& \leq a_{0}\left|u_{n}-u\right|_{1}+\left.\left.c_{6}|| u_{n}\right|^{\alpha(x)-1}\right|_{\alpha^{\prime}(x)} \cdot\left|u_{n}-u\right|_{\alpha(x)} .
\end{aligned}
$$


It is immediate that $\left|u_{n}-u\right|_{1} \rightarrow 0$, that $\left.\left.|| u_{n}\right|^{\alpha(x)-1}\right|_{\alpha^{\prime}(x)}$ is bounded and that $\left|u_{n}-u\right|_{\alpha(x)} \rightarrow 0$. Hence

$$
\int_{\Omega} f\left(x, u_{n}\right)\left(u_{n}-u\right) d x \rightarrow 0 .
$$

Similarly,

$$
\int_{\Omega} f(x, u)\left(u_{n}-u\right) d x \rightarrow 0 .
$$

Thus

$$
\int_{\Omega}\left(\left|\nabla u_{n}\right|^{p(x)-2} \nabla u_{n}-|\nabla u|^{p(x)-2} \nabla u\right) \nabla\left(u_{n}-u\right) d x \rightarrow 0
$$

as $n \rightarrow \infty$. Similar to Theorem 3.1 in [3], we divide $\Omega$ into two parts:

$$
\Omega_{1}=\{x \in \Omega: p(x)<2\}, \quad \Omega_{2}=\{x \in \Omega: p(x) \geq 2\} .
$$

On $\Omega_{1}$, we have

$$
\begin{aligned}
\int_{\Omega_{1}} \mid \nabla u_{n} & -\left.\nabla u\right|^{p(x)} d x \\
\leq & c_{7} \int_{\Omega_{1}}\left(\left(\left|\nabla u_{n}\right|^{p(x)-2} \nabla u_{n}-|\nabla u|^{p(x)-2} \nabla u\right)\left(\nabla u_{n}-\nabla u\right)\right)^{p(x) / 2} \\
& \times\left(\left|\nabla u_{n}\right|^{p(x)}+|\nabla u|^{p(x)}\right)^{(2-p(x)) / 2} d x \\
\leq & c_{8}\left|\left(\left(\left|\nabla u_{n}\right|^{p(x)-2} \nabla u_{n}-|\nabla u|^{p(x)-2} \nabla u\right)\left(\nabla u_{n}-\nabla u\right)\right)^{p(x) / 2}\right|_{2 / p(x), \Omega_{1}} \\
& \times\left|\left(\left|\nabla u_{n}\right|^{p(x)}+|\nabla u|^{p(x)}\right)^{(2-p(x)) / 2}\right|_{2 /(2-p(x)), \Omega_{1}} .
\end{aligned}
$$

Noting that

$$
\int_{\Omega_{1}}\left(\left|\nabla u_{n}\right|^{p(x)-2} \nabla u_{n}-|\nabla u|^{p(x)-2} \nabla u\right) \nabla\left(u_{n}-u\right) d x \rightarrow 0
$$

implies

$$
\left|\left(\left(\left|\nabla u_{n}\right|^{p(x)-2} \nabla u_{n}-|\nabla u|^{p(x)-2} \nabla u\right)\left(\nabla u_{n}-\nabla u\right)\right)^{p(x) / 2}\right|_{2 / p(x), \Omega_{1}} \rightarrow 0,
$$

we have $\int_{\Omega_{1}}\left|\nabla u_{n}-\nabla u\right|^{p(x)} d x \rightarrow 0$. On $\Omega_{2}$, we have

$$
\begin{aligned}
& \int_{\Omega_{2}}\left|\nabla u_{n}-\nabla u\right|^{p(x)} d x \\
& \quad \leq c_{9} \int_{\Omega_{2}}\left(\left|\nabla u_{n}\right|^{p(x)-2} \nabla u_{n}-|\nabla u|^{p(x)-2} \nabla u\right)\left(\nabla u_{n}-\nabla u\right) d x \rightarrow 0 .
\end{aligned}
$$

Thus $\int_{\Omega}\left|\nabla u_{n}-\nabla u\right|^{p(x)} d x \rightarrow 0$. Now it is easy to get $u_{n} \rightarrow u$ in $W_{0, G}^{1, p(x)}(\Omega)$ as $n \rightarrow \infty$.

LEMma 3.3. There exists $R_{n}>0$ such that $\widetilde{\varphi}(u) \leq 0$ for any $u \in Y_{n}$ with $\|u\| \geq R_{n}$. 
Proof. For any $u \in Y_{n}$, we have

$$
\begin{aligned}
\widetilde{\varphi}(u) & =\int_{\Omega}\left(\frac{|\nabla u|^{p(x)}+|u|^{p(x)}}{p(x)}-\frac{|u|^{p^{*}(x)}}{p^{*}(x)}-F(x, u)\right) d x \\
& \leq \int_{\Omega}\left(\frac{|\nabla u|^{p(x)}+|u|^{p(x)}}{p_{-}}-\frac{|u|^{p^{*}(x)}}{p_{+}^{*}}+a_{0}|u|+a_{1} \frac{|u|^{\alpha(x)}}{\alpha(x)}\right) d x .
\end{aligned}
$$

Noting that $\alpha(x) \ll p^{*}(x)$, by the Young inequality, for any $\varepsilon_{5} \in(0,1)$, we get

$$
a_{0}|u|+a_{1} \frac{|u|^{\alpha(x)}}{\alpha(x)} \leq \varepsilon_{5}|u|^{p^{*}(x)}+c_{10} .
$$

Let $\varepsilon_{5}$ be so small that $\varepsilon_{5} \leq 1 / 2 p_{+}^{*}$. Then

$$
\widetilde{\varphi}(u) \leq \int_{\Omega}\left(\frac{|\nabla u|^{p(x)}+|u|^{p(x)}}{p_{-}}-\frac{|u|^{p^{*}(x)}}{2 p_{+}^{*}}+c_{10}\right) d x .
$$

In the following, we will consider $\int_{\Omega}\left(\frac{|\nabla u|^{p(x)}+|u|^{p(x)}}{p_{-}}-\frac{|u|^{p^{*}(x)}}{2 p_{+}^{*}}\right) d x$.

Let $\|u\|>1$. Note that $p, p^{*} \in C(\bar{\Omega})$ and $p(x) \ll p^{*}(x)$. For any $x \in \bar{\Omega}$, there exists an open subset $Q(x)$ of $\bar{\Omega}$ such that

$$
p_{x}=\sup _{y \in Q(x)} p(y)<p_{x}^{*}=\inf _{y \in Q(x)} p^{*}(y) .
$$

Then $\{Q(x)\}_{x \in \bar{\Omega}}$ is an open covering of $\bar{\Omega}$. As $\bar{\Omega}$ is compact, there is a finite subcovering $\left\{Q\left(x_{i}\right)\right\}_{i=1}^{k}$. We can use all the hyperplanes, for each of which there exists at least one hypersurface of some $\left\{Q\left(x_{i}\right)\right\}$ lying on it, to divide $\bigcup_{i=1}^{k} Q\left(x_{i}\right)$ into finitely many open hypercubes $\left\{\Omega_{i}\right\}_{i=1}^{m}$ which are pairwise disjoint. It is obvious that $\bar{\Omega}=\bigcup_{i=1}^{m} \bar{\Omega}_{i}$ and

$$
p_{i+}=\sup _{x \in \Omega_{i}} p(x)<p_{i-}^{*}=\inf _{x \in \Omega_{i}} p^{*}(x)
$$

for $i=1, \ldots, m$. Denote $r_{i}=\|u\|_{\Omega_{i}}$. Then

$$
\begin{aligned}
\int_{\Omega}\left(\frac{|\nabla u|^{p(x)}+|u|^{p(x)}}{p_{-}}-\frac{|u|^{p^{*}(x)}}{2 p_{+}^{*}}\right) d x & \\
& =\sum_{i=1}^{m} \int_{\Omega_{i}}\left(\frac{|\nabla u|^{p(x)}+|u|^{p(x)}}{p_{-}}-\frac{|u|^{p^{*}(x)}}{2 p_{+}^{*}}\right) d x \\
& =\sum_{r_{i}>1} \int_{\Omega_{i}}\left(\frac{|\nabla u|^{p(x)}+|u|^{p(x)}}{p_{-}}-\frac{|u|^{p^{*}(x)}}{2 p_{+}^{*}}\right) d x
\end{aligned}
$$




$$
\begin{aligned}
& +\sum_{r_{i} \leq 1} \int_{\Omega_{i}}\left(\frac{|\nabla u|^{p(x)}+|u|^{p(x)}}{p_{-}}-\frac{|u|^{p^{*}(x)}}{2 p_{+}^{*}}\right) d x \\
\leq & \sum_{r_{i}>1}\left(\frac{\|u\|_{\Omega_{i}}^{p_{i+}}}{p_{-}}-\frac{k_{n_{i}}}{2 p_{+}^{*}}\|u\|_{\Omega_{i}}^{p_{i-}^{*}}\right)+\frac{m}{p_{-}},
\end{aligned}
$$

where $k_{n_{i}}=\inf _{\left.u \in Y_{n}\right|_{\Omega_{i}},\|u\|_{\Omega_{i}}=1} \int_{\Omega_{i}}|u|^{p^{*}(x)} d x$. As $\left.Y_{n}\right|_{\Omega_{i}}$ is a finite-dimensional space, we have $k_{n_{i}}>0$ for $i=1, \ldots, m$.

We denote by $s_{i}$ the maximum of the polynomial $\frac{t^{p_{i+}}}{p_{-}}-\frac{k_{n_{i}}}{2 p_{+}^{*}} t_{i-}^{p_{i-}^{*}}$ on $[0, \infty)$ for $i=1, \ldots, m$. Then there exists $t_{0}>1$ such that

$$
\frac{t^{p_{i+}}}{p_{-}}-\frac{k_{n_{i}}}{2 p_{+}^{*}} t^{p_{i-}^{*}}+c_{11} \leq 0
$$

for $t>t_{0}$ and $i=1, \ldots, m$, where $c_{11}=\sum_{i=1}^{m} s_{i}+m / p_{-}+c_{10}$ meas $\Omega$.

Let $R_{n} \geq m^{2} t_{0}$. If $\|u\| \geq R_{n}$, then $\|u\| \geq m^{2} t_{0}>1$. It is easy to verify that there exists at least one $i_{0}$ such that $\|u\| \Omega_{i_{0}} \geq t_{0}>1$, thus

$$
\widetilde{\varphi}(u) \leq \frac{\|u\|_{\Omega_{i_{0}}}^{p_{i_{0}}}}{p_{-}}-\frac{k_{n_{i_{0}}}}{2 p_{+}^{*}}\|u\|_{\Omega_{i_{0}}}^{p_{i_{0}-}^{*}}+c_{11} \leq 0 .
$$

Now we get the result.

LEMMA 3.4. There exist $r_{n}>0$ and $a_{n} \rightarrow \infty(n \rightarrow \infty)$ such that $\widetilde{\varphi}(u) \geq a_{n}$ for any $u \in Z_{n}$ with $\|u\|=r_{n}$.

Proof. For $u \in Z_{n}$. Let $\|u\|>1$, By condition (H1), we get

$$
\begin{aligned}
\widetilde{\varphi}(u) & =\int_{\Omega}\left(\frac{|\nabla u|^{p(x)}+|u|^{p(x)}}{p(x)}-\frac{|u|^{p^{*}(x)}}{p^{*}(x)}-F(x, u)\right) d x \\
& \geq \int_{\Omega}\left(\frac{|\nabla u|^{p(x)}+|u|^{p(x)}}{p_{+}}-\frac{|u|^{p^{*}(x)}}{p^{*}(x)}-a_{0}|u|-a_{1} \frac{|u|^{\alpha(x)}}{\alpha(x)}\right) d x \\
& \geq \int_{\Omega}\left(\frac{|\nabla u|^{p(x)}+|u|^{p(x)}}{p_{+}}-c_{12}|u|^{p^{*}(x)}\right) d x-c_{13} .
\end{aligned}
$$

Denote

$$
\theta_{n}=\sup _{u \in Z_{n},\|u\| \leq 1} \int_{\Omega}|u|^{p^{*}(x)} d x
$$

thus

$$
\widetilde{\varphi}(u) \geq \frac{\|u\| \|_{-}^{p_{-}}}{p_{+}}-c_{12} \theta_{n}\|u\|^{p_{+}^{*}}-c_{13} .
$$

Let

$$
r_{n}=\max \left\{1,\left(\frac{p_{-}}{c_{12} p_{+} p_{+}^{*} \theta_{n}}\right)^{\frac{1}{p_{+}^{*}-p_{-}}},\left(\frac{2 c_{13} p_{+} p_{+}^{*}}{p_{+}^{*}-p_{-}}\right)^{\frac{1}{p_{-}}}\right\} .
$$


By Lemma 2.2, we deduce that $\theta_{n} \rightarrow 0$ as $n \rightarrow \infty$; then

$$
\widetilde{\varphi}(u) \geq r_{n}^{p_{-}} \frac{p_{+}^{*}-p_{-}}{p_{+} p_{+}^{*}}-c_{13} \triangleq a_{n},
$$

when $n$ is sufficiently large and $\|u\|=r_{n}$. It is easy to conclude that $a_{n} \rightarrow \infty$ as $n \rightarrow \infty$.

Proof of Theorem 1.1. By condition (H2), $\widetilde{\varphi}$ is an even functional on $W_{0, O(N)}^{1, p(x)}(\Omega)$. By Lemmas 3.1-3.4 and the fountain theorem in [14, we know that the functional $\widetilde{\varphi}$ has a sequence of critical points $u_{k} \subset W_{0, O(N)}^{1, p(x)}(\Omega)$ such that $\varphi\left(u_{k}\right)=\widetilde{\varphi}\left(u_{k}\right)=c_{k} \rightarrow \infty$ as $k \rightarrow \infty$. This completes the proof.

Acknowledgements. This research was supported by Science Research Foundation in Harbin Institute of Technology (HITC200702) and The Natural Science Foundation of Heilongjiang Province (A2007-04).

\section{References}

[1] C. O. Alves and M. A. S. Souto, Existence of solutions for a class of problems in $\mathbb{R}^{N}$ involving $p(x)$-Laplacian, in: Progr. Nonlinear Differential Equations Appl. 66, Birkhäuser, 2005, 17-32.

[2] S. Antontsev, M. Chipot and Y. Xie, Uniquenesss results for equations of the $p(x)$ Laplacian type, Adv. Math. Sci. Appl. 17 (2007), 287-304.

[3] J. Chabrowski and Y. Fu, Existence of solutions for $p(x)$-Laplacian problems on a bounded domain, J. Math. Anal. Appl. 306 (2005), 604-618; Erratum, ibid. 323 (2006), 1483.

[4] D. Edmunds, J. Lang and A. Nekvinda, On $L^{p(x)}$ norms, Proc. Roy. Soc. London Ser. A 455 (1999), 219-225.

[5] D. Edmunds and J. Rákosník, Sobolev embeddings with variable exponent, Studia Math. 143 (2000), 267-293.

[6] X. Fan and X. Han, Existence and multiplicity of solutions for $p(x)$-Laplacian equations in $\mathbb{R}^{N}$, Nonlinear Anal. 59 (2004), 173-188.

[7] X. Fan, J. Shen and D. Zhao, Sobolev embedding theorems for spaces $W^{k, p(x)}(\Omega)$, J. Math. Anal. Appl. 262 (2001), 749-760.

[8] X. Fan, Y. Zhao and D. Zhao, Compact imbedding theorems with symmetry of Strauss-Lions type for the space $W^{1, p(x)}(\Omega)$, J. Math. Anal. Appl. 255 (2001), 333348.

[9] Y. Q. Fu, The principle of concentration compactness in $L^{p(x)}$ spaces and its application, Nonlinear Anal. 71 (2009), 1876-1892.

[10] O. Kováčik and J. Rákosník, On spaces $L^{p(x)}$ and $W^{k, p(x)}$, Czechoslovak Math. J. 41 (1991), 592-618.

[11] W. Krawcewicz and W. Marzantowicz, Some remarks on the Lusternik-Schnirelman method for non-differentiable functionals invariant with respect to a finite group action, Rocky Mountain J. Math. 20 (1990), 1041-1049.

[12] P.-L. Lions, The concentration-compactness principle in the calculus of variations, the limit case, Part I, Rev. Mat. Iberoamer. 1 (1985), no. 1, 145-201; Part II, ibid., 1 (1985), no. 2, 45-121. 
[13] M. Mihăilescu and V. Rădulescu, A multiplicity result for a nonlinear degenerate problem arising in the theory of electrorheological fluids, Proc. Roy. Soc. London Ser. A 462 (2006), 2625-2641.

[14] M. Willem, Minimax Theorems, Birkhäuser, Boston, MA, 1996.

[15] X. Zhang and Y. Q. Fu, Solutions for a class of hemivariational inequalities with $p(x)$-Laplacian, Ann. Polon. Math. 95 (2009), 273-288.

Xing Zhang, Xia Zhang (corresponding author), Yongqiang Fu

Department of Mathematics

Harbin Institute of Technology

Harbin 150001, China

E-mail: piecesummer1984@163.com

Received 27.5.2009

and in final form 1.9.2009 